\title{
Der Erwerb zusätzlicher Facharzttitel wird günstiger
}

C. Hänggeli, Geschäftsleiter Sekretariat Aus-, Weiterund Fortbildung (AWF)
Für Ärztinnen und Ärzte in Weiterbildung gibt es eine gute und eine schlechte Nachricht. Die schlechte zuerst: Aufgrund der Finanzsituation der FMH hat die Ärztekammer am 3. Mai 2007 beschlossen, die sogenannten Rückerstattungen von Mitgliederbeiträgen nach dem Erwerb des Facharzttitels aufzuheben. Diese Massnahme entspricht einem von vier Modulen, die der langfristigen Budgetstabilisierung der FMH dienen. Die gute Nachricht: Ärztinnen und Ärzte, die nach dem Erwerb des ersten Facharzttitels einen zweiten oder dritten Titel erwerben, bezahlen ab dem 1. Januar 2008 Fr. 1500.- statt Fr. 2500.-. In Anbetracht der gesunden finanziellen Situation des Bereichs AWF [1] hat die KWFB die Gebührenreduktion einstimmig gutgeheissen.

\section{Rückblick}

Seit der Inkraftsetzung des Personenfreizügigkeitsabkommens mit der EU und des Freizügigkeitsgesetzes (FMPG) per 1. Juni 2002 erteilt die FMH eidgenössische Facharzttitel. Mit dem gleichzeitigen Wegfall der Mitgliedschaftspflicht für den Erwerb und die Führung eines Facharzttitels musste der ganze Weiter- und Fortbildungsbereich zwingend über kostendeckende Diplomgebühren finanziert werden. Andernfalls hätten sich Nichtmitglieder ihren Facharzttitel von den Mitgliedern finanzieren lassen - eine typische Trittbrettfahrersituation, die keine Option darstellte. Die im Jahr 2001 berechnete Gebühr von Fr. 4000.- pro Facharzttitel erweist sich bei längerfristiger Betrachtung als korrekt. Vor 2002 erteilte die FMH im langjährigen Mittel etwa 800 Facharzttitel pro Jahr (Tab. und Abb. 1). Dank des kurzfristigen Anstiegs der Titelerteilungen in den letzten Jahren konnte das Sekretariat AWF Reserven bilden, die in Zukunft für Projekte zur Steigerung der Weiterbildungsqualität zur Verfügung stehen.

\section{FMH-interne Solidarität mit der jungen Ärztegeneration}

4000 Franken für einen Facharzttitel sind viel Geld. Bei der Beratung der Titelgebühr hat sich die Ärztekammer 2001 vom Gedanken leiten lassen, die generationenübergreifende Solidarität unter den FMH-Mitgliedern beizubehalten und gleichzeitig einen Anreiz für den Beitritt zur FMH zu schaffen. FMH-Mitglieder sollten bei Erwerb des Facharzttitels den grössten Teil ihrer bereits bezahlten Mitgliederbeiträge zurückerhalten und zusätzlich in den Genuss einer Beitragsreduktion für die nächsten fünf Jahre kom-

Tabelle 1

Erteilte Weiterbildungstitel 1996-2006.

\begin{tabular}{lcccc} 
Jahr & Facharzttitel & Schwerpunkte & Praktischer Arzt & Total \\
\hline 1996 & 766 & 0 & 4 & 770 \\
\hline 1997 & 965 & 0 & 49 & 1014 \\
\hline 1998 & 805 & 35 & 88 & 928 \\
\hline 1999 & 873 & 89 & 112 & 1074 \\
\hline 2000 & 805 & 322 & 218 & 1345 \\
\hline 2001 & 776 & 154 & 142 & 1072 \\
\hline 2002 & 1609 & 119 & 473 & 2201 \\
\hline 2003 & 1551 & 472 & 254 & 2277 \\
\hline 2004 & 1140 & 319 & 120 & 1579 \\
\hline 2005 & 1039 & 186 & 135 & 1360 \\
\hline 2006 & 958 & 177 & 134 & 1269 \\
\hline
\end{tabular}


Abbildung 1

Erteilte Weiterbildungstitel 1996-2006.



men. Diese Regelung, wonach FMH-Mitglieder faktisch Fr. 2000.- statt Fr. 4000.- für den Facharzttitel bezahlt haben, ist jetzt von der Ärztekammer modifiziert worden. Inskünftig gibt es nur noch eine Rückzahlung von Mitgliederbeiträgen in der Höhe von Fr. 1000.- beim Erwerb des ersten Facharzttitels. Ärztinnen und Ärzte, die das Arztdiplom vor 2007 erworben haben, sind davon nicht betroffen und können noch bis 2013 Rückzahlungen im Umfang von Fr. 2000.beanspruchen. Die Ärztekammer kann überdies gemäss ihrem Beschluss aus dem Jahr 2003 weiterhin aus allgemeinen Mitteln Weiterbildungsprojekte unterstützen, wenn die finanzielle Situation dies erfordert.

\section{Die FMH-Mitgliedschaft zahlt sich aus!}

Ärztinnen und Ärzte, die ab dem ersten Weiterbildungsjahr der FMH beitreten, profitieren in jedem Fall: Einerseits erhalten sie die Mitgliederbeiträge beim Erwerb des Facharzttitels zurückerstattet, anderseits sind sämtliche Auskünfte rund um die Weiterbildung (einfache Anfragen, Erstellung eines Weiterbildungsplanes usw.) kostenlos.

\section{Franken für ein Stück Papier?}

Ein oft gehörter Vorwurf sei an dieser Stelle richtiggestellt: Die Diplomgebühr dient nicht allein der Überprüfung des Titelgesuches und der Ab- gabe der Diplomurkunde. Der grösste Teil des Aufwands im Bereich Weiter- und Fortbildung betrifft die Regelung und Verbesserung der Weiterbildungsqualität. Dazu gehören zum Beispiel: - die Entschädigung aller im Bereich Weiterund Fortbildung tätigen Organe und Kommissionen;

- die gesamtschweizerische Umfrage bei allen Assistenzärztinnen und Assistenzärzten zur Qualität ihrer Weiterbildungsstätte;

- die Akkreditierung der Weiterbildungsprogramme durch das Eidgenössische Departement des Innern (EDI);

- die Zertifizierung aller Weiterbildungsstätten (Spitäler, Kliniken, Abteilungen) in der Schweiz;

- Assessmentprojekte (Mini-CEX, Selbstevaluation).

Mit der Führung des Sekretariats Aus-, Weiterund Fortbildung (AWF) als finanziell eigenständiges Service-Center ist sichergestellt, dass sämtliche Aufwendungen und Erträge von der allgemeinen FMH-Rechnung klar abgegrenzt sind und die Finanzierung dieses Bereiches der Öffentlichkeit und den Behörden gegenüber transparent ausgewiesen werden kann. Die detaillierten Projekte und Zahlen sind den jährlichen Tätigkeitsberichten des Sekretariates AWF zu entnehmen [1]. 


\section{Was ist neu in der Gebührenordnung?}

Aufgrund des Wegfalls der Rückzahlungen von Mitgliederbeiträgen nach Erwerb des Facharzttitels hat die KWFB den Erwerb eines zweiten oder dritten Facharzttitels von Fr. 2500.- auf Fr. 1500.- reduziert. Mit dieser Massnahme wird der Erwerb von zusätzlichen Facharzttiteln erleichtert. Die Gebühr von Fr. 4000.- für den ersten Titel spiegelt als «Grundgebühr» die gesamten Kosten des Bereichs AWF. Zusätzliche Titel sollen im wesentlichen die Kosten für das Titelerteilungsverfahren wiedergeben. Die übrigen Änderungen sind eher redaktioneller Natur und aus Gründen der Vollständigkeit und Klarheit vorgenommen worden (u.a. Gebühren der Ein- sprachekommissionen, mehrwertsteuerpflichtige Leistungen, Übergangsbestimmungen).

\section{Ausblick}

Obwohl in den nächsten Jahren verschiedene kostenintensive Projekte in die Realisierungsphase treten, ist die Finanzierung mit der aktuellen Gebührenordnung sichergestellt. Eine über viele Jahre konstante Titelgebühr dient der rechtsgleichen Behandlung aller Titelerwerber und stärkt das Vertrauen in die FMH. Jährliche Preissteigerungen stellen im Gesundheitswesen den Normalfall dar. Nicht so im Sekretariat AWF. Auch das ist eine gute Nachricht!

\section{Gebührenordnung}

Diese Gebührenordnung ersetzt diejenige aus dem Jahr 2002 [2].

Gestützt auf Art. 69 WBO hat die KWFB am 6. September 2007

folgende Gebührenordnung beschlossen:

\section{Erteilung eidgenössischer Weiterbildungstitel}

Erster Facharzttitel

Fr. 4000.-

Exklusiv für Mitglieder der FMH

Rückzahlung von Mitgliederbeiträgen: bis zu Fr. 200.- pro Mitgliedschaftsjahr für maximal 5 Jahre beim Erwerb des ersten Facharzttitels (maximal Fr. 1000.-).

Zweiter und jeder weitere Facharzttitel

Fr. 1500.(ausländische Titel werden nicht berücksichtigt)

Gebühr für die Facharzt- bzw. Schwerpunktprüfung

Die Gebühr wird von der zuständigen Fachgesellschaft festgelegt.

\section{Erteilung von FMH-Weiterbildungstiteln (exklusiv an Mitglieder der FMH)}

Facharzttitel (Handchirurgie und Neuropathologie)

Rückzahlungen analog Ziffer 1

Schwerpunkt

Fähigkeitsausweise

Die Gebühr wird von der zuständigen Gesellschaft festgelegt.

\section{Dienstleistungen im Bereich Weiterbildungstitel}

Schriftliche Auskünfte durch das Sekretariat AWF

geringer Aufwand (1/2-1 Stunde)

mittlerer Aufwand (1-2 Stunden)

grosser Aufwand (über 2 Stunden)
Fr. 4000.-

Fr. 1000.-
Mitglieder Nichtmitglieder

kostenlos

Fr. 100.-*

kostenlos Fr. 200.- ${ }^{*}$

kostenlos Fr. 300.-* 
Erstellung eines Weiterbildungsplanes durch die Titelkommission geringer Aufwand (1/2-1 Stunde)

Mitglieder Nichtmitglieder

mittlerer Aufwand (1-2 Stunden)

kostenlos

Fr. 200.-*

grosser Aufwand (über 2 Stunden)

kostenlos

Fr. 300.-*

Ausstellung einer Äquivalenzbestätigung (nur für Ärztinnen

kostenlos

Fr. 400.- ${ }^{\star}$ und Ärzte mit nicht anerkanntem Arztdiplom)

Erstellung eines Duplikates für abhanden gekommene Diplome

Fr. 1000.-

Weiterverrechnung Beglaubigung

Fr. 150.- ${ }^{*}$

Zertifizierung von Fähigkeitsausweisen

Fr. 20.-*

Datenverwaltung von Fähigkeitsausweisen

Fr. 1000.-*

Fr. 300.-*

\section{Dienstleistungen im Bereich Fortbildung}

Erteilung Label FMH-approved (Art. 7 FBO; vgl. Kriterien für die Anerkennung auf www.fmh.ch)

Erstbeurteilung

Fr. 350.-*

wiederholte Veranstaltung

Fr. $150 .-^{*}$

\section{Dienstleistungen im Bereich Weiterbildungsstätten}

\section{Visitationsgebühren}

grosse Kliniken

Fr. 5000.-

kleine Kliniken (1 bis 3 Assistenten)

Fr. 4000.-

Für Visitationen, bei denen mehrere Weiterbildungsstätten (z. B. Facharzttitel und Schwerpunkte) und/oder eine Weiterbildungsstätte mit mehreren Anerkennungen visitiert werden, legt das Büro KWFB die Visitationsgebühr im Einzelfall fest.

\section{Gebühren der Einsprachekommissionen}

Verfahrenskosten für Einspracheverfahren je nach Aufwand

Fr. 500.- bis 1500.-.

\section{7. Übergangs- und Ergänzungs- bestimmungen}

1. Die Titelgebühr wird mit der Titelerteilung in Rechnung gestellt, die innert 30 Tagen zu bezahlen ist. Das Diplom wird erst nach Zahlungseingang zugestellt.

2. Bereits bezahlte Gebühren für den Weiterbildungstitel «praktischer Arzt / praktische Ärztin» oder für die Äquivalenzbestätigung werden beim Erwerb des Facharzttitels angerechnet.

3. Die Reduktion von Mitgliederbeiträgen nach Erwerb des ersten Facharzttitels wird bis zum Jahr 2013 weitergeführt, allerdings nur für FMH-Mitglieder, die ihr Arztdiplom vor dem 1. Januar 2007 erworben haben.

4. Wer die FMH-Weiterbildungstitel Handchirurgie und Neuropathologie nach altem
Fr. 1000.- (wie für den früheren Schwerpunkt). Pioniere erwerben den Titel nach neuem Programm.

5. Die neue Gebühr für einen zweiten Facharzttitel gilt ab 1. Januar 2008 (Anerkennungsdatum).

6. Die Übergangs- und Ergänzungsbestimmungen der alten Gebührenordnung aus dem Jahr 2002 bleiben weiterhin gültig, soweit sie noch anwendbar sind.

\section{Literatur}

1 Giger M, Hänggeli C. Tätigkeitsbericht 2006 der Kommission für Weiter- und Fortbildung (KWFB) / Sekretariat AWF. Schweiz Ärztezeitung. 2007; 88(23):980-98.

2 Hänggeli C. Wie bitte? 4000 Franken für einen eidgenössischen Facharzttitel? Schweiz Ärztezeitung. 2002;83(23):1167-70. 